\title{
Potential of High-Frequency EPR for Investigation of Supported Vanadium Oxide Catalysts
}

\author{
Arne Dinse, ${ }^{\dagger}$ Andrzej Ozarowski, ${ }^{\ddagger}$ Christian Hess,,${ }^{\S, \|}$ Reinhard Schomäcker, ${ }^{\dagger}$ and \\ Klaus-Peter Dinse*,\$ \\ Department of Chemistry, Technical University of Berlin, Strasse des 17, Juni 124, D-10623 Berlin, Germany, \\ National High Magnetic Field Laboratory, 1800 East Paul Dirac Drive, Tallahassee, Florida 32310-3706, \\ Eduard-Zintl-Institut für Anorganische and Physikalische Chemie, Technische Universität Darmstadt, \\ Petersenstrasse 20, D-64287 Darmstadt, Germany, and Department of Inorganic Chemistry, \\ Fritz-Haber-Institute of the Max Planck Society, Faradayweg 4-6, 14195 Berlin, Germany
}

Received: August 11, 2008; Revised Manuscript Received: September 8, 2008

\begin{abstract}
Supported vanadium oxide catalysts were studied by high-frequency EPR for determination of the paramagnetic vanadium oxidation states vanadium(IV) and vanadium(III). $\mathrm{TiO}_{2^{-}}, \gamma-\mathrm{Al}_{2} \mathrm{O}_{3^{-}}$, and $\mathrm{SiO}_{2}$ (SBA-15)-supported catalysts were investigated before and after being used for the oxidative dehydrogenation of propane. While the SBA-15-supported catalyst exhibits only one distinct position of vanadium species with the oxidation state IV, $\mathrm{Al}_{2} \mathrm{O}_{3}$ reveals a distribution of vanadium(IV) sites. For this support material formation of oxoradicals has been observed, indicating its capability for trapping electrons during the catalytic cycle. The $\mathrm{TiO}_{2}$-supported material apparently does not stabilize vanadium(IV), which can be attributed to either a high mobility of existing oxygen vacancies or the competitive capability of titanium(IV) surface states to trap electrons. Titanium in its oxidation state of vanadium(III) and surface-trapped $\mathrm{O}_{2}{ }^{(-)}$species were identified as potential electron sinks in the case of the $\mathrm{TiO}_{2}$-supported catalyst. The increase in catalytic activity in the order of SBA-15< $\mathrm{Al}_{2} \mathrm{O}_{3}<\mathrm{TiO}_{2}$ therefore could be caused by different reduction mechanisms depending on the chosen support material. No vanadium in its oxidation state III was detected in any of the samples, which indicates that such species are either short lived or not existent during the oxidative dehydrogenation of propane.
\end{abstract}

\section{Introduction}

The oxidative dehydrogenation of propane (ODP) on supported vanadia catalysts is an example of a selective oxidation reaction of low hydrocarbons and an important probe reaction for the fundamental understanding of heterogeneous catalysis on a molecular basis. Many experimental and theoretical approaches to this subject have been performed in the past decades. ${ }^{1-6}$ However, the key factors for control of the catalytic performance of this reaction are still not fully understood. Supported vanadia catalysts offer a high catalytic activity, but in the case of ODP the similarity of reactants and products leads to poor selectivity. ${ }^{7}$ Analytical investigations indicate that monomeric and/or associated vanadium species are present on the surface of the support materials studied here. ${ }^{8,9}$ The generally accepted reaction network ${ }^{10}$ of ODP is depicted in Figure 1.

Propane reacts to carbon oxides and propene, which can subsequently also react to carbon oxides. Kinetic studies, ${ }^{11,12}$ however, indicate that direct oxidation of propane to carbon oxides occurs only to a small extent.

For the dehydrogenation of propane to propene, theoretical DFT calculations by Rozanska et al. ${ }^{6}$ for silica-supported vanadium oxide catalysts and Gilardoni et al. ${ }^{4}$ for $\mathrm{V}_{2} \mathrm{O}_{5}$ propose the reaction mechanism depicted in Figure 2. On the basis of the lower activation barriers, a one-electron reduction (vanadi$\mathrm{um}(\mathrm{V})$ to vanadium(IV)) was found to be more likely than a two-electron reduction (vanadium(V) to vanadium(III)). The

* To whom correspondence should be addressed. Phone: +49-6151162607. Fax: +49-6151-164347. E-mail: dinse@chemie.tu-darmstadt.de.

Technical University of Berlin.

* National High Magnetic Field Laboratory.

$\S$ Technische Universität Darmstadt.

"Fritz-Haber-Institute of the Max Planck Society.

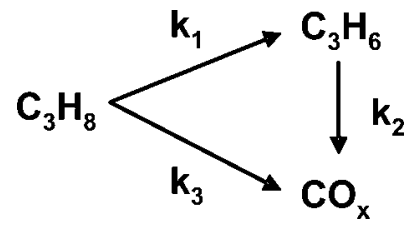

Figure 1. Reaction network of ODP.

depicted mechanisms are also consistent with isotopic tracer experiments done by Chen et al. ${ }^{13}$ All studies conclude that the $\mathrm{C}-\mathrm{H}$ bond abstraction of the secondary hydrogen atom is the rate-determining step followed by a second hydrogen transfer and formation of water. The mentioned investigations also lead to the conclusion that the vanadyl oxygen, which is often denoted as lattice oxygen, is the main active site for the reaction. For the consecutive oxidation of propene, it is assumed that $\mathrm{C}-\mathrm{H}$ bond cleavage is also the rate-determining step and carbon oxide species form under further participation of lattice oxygen. A study by Chen et al. ${ }^{14}$ suggested that the catalytic activity is primarily influenced by the reducibility of the catalyst, which is a measure for the ability to delocalize electrons during a catalytic turn over. The increase in reducibility with increasing vanadium loading may be explained by formation of active polyvanadate species on the support surface which facilitate electron delocalization and lead to an increase in the rate constants $k_{1}, k_{2}$, and $k_{3}$. Since a change in reducibility affects the rate constants to different extents, ${ }^{15}$ it also explains a change in the selectivity toward propene, which is described by the ratio of $k_{3}$ to $k_{1}$. In the case of silica as a support material, the presence of dimeric and/or polymeric species at low loadings has only recently been observed for SBA-15-supported vanadium oxide. ${ }^{9}$ At very high loadings, activity and selectivity decrease because of formation of crystalline $\mathrm{V}_{2} \mathrm{O}_{5}$. 


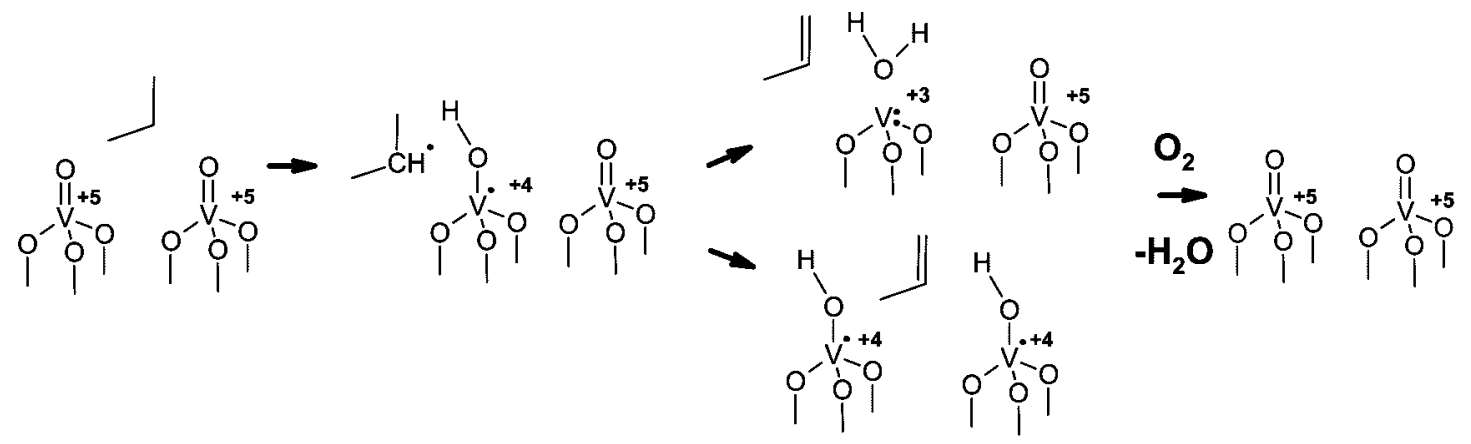

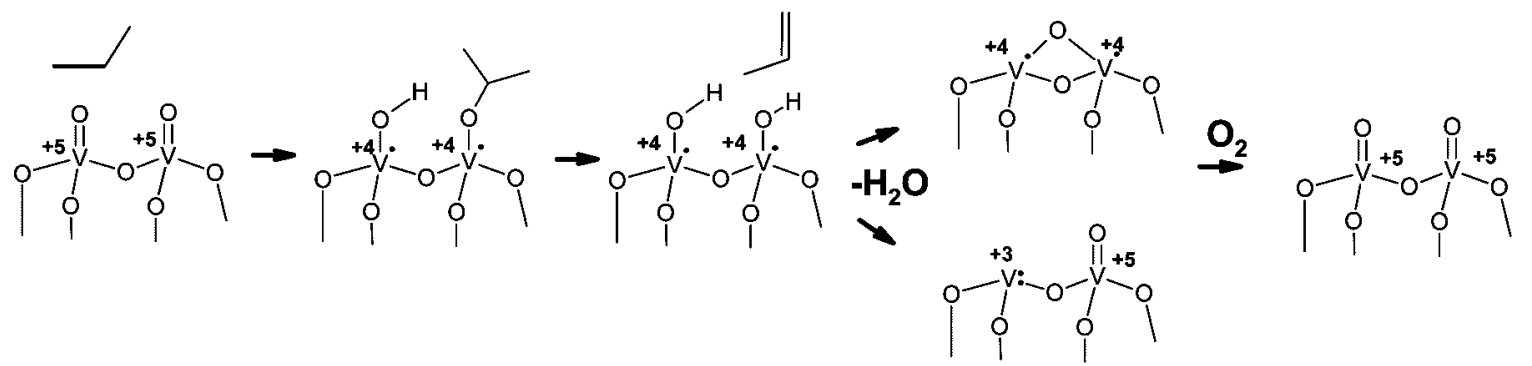

Figure 2. Reaction mechanisms of ODP as proposed by Rozanska et al. ${ }^{6}$ for monomeric vanadium surface species (A) and Chen et al. ${ }^{14}$ for associated vanadium surface species (B), respectively.

TABLE 1: Parameters during Reaction Before Samples Were Quenched with Nitrogen and Sealed in a Pseudo-in-Situ State

\begin{tabular}{|c|c|c|c|c|c|c|c|c|c|c|}
\hline catalyst & $m, \mathrm{mg}$ & $T,{ }^{\circ} \mathrm{C}$ & density, $\mathrm{V} \mathrm{nm}{ }^{-2}$ & wt $\%, \% \mathrm{~V}_{2} \mathrm{O}_{5}$ & BET, $\mathrm{m}^{2} \mathrm{~g}^{-1}$ & feed $\mathrm{C}_{3} \mathrm{H}_{8}: \mathrm{O}_{2}$ & flow, $\mathrm{cm}^{3} \mathrm{~s}^{-1}$ & $X_{\mathrm{C} 3 \mathrm{H} 8}, \%$ & $X_{\mathrm{O} 2}, \%$ & $S_{\mathrm{C} 3 \mathrm{H} 6}, \%$ \\
\hline $\mathrm{V}-\mathrm{SBA}-15$ & 250 & 500 & 0.7 & 4.7 & 533 & $2: 4$ & 0.4 & 25 & 40 & 21 \\
\hline V-SBA-15 & 250 & 500 & 0.7 & 4.7 & 533 & $8: 1$ & 1 & 4 & 63 & 57 \\
\hline $\mathrm{V}-\mathrm{Al}_{2} \mathrm{O}_{3}$ & 150 & 450 & 1.4 & 2.1 & 96 & $2: 4$ & 1 & 18 & 24 & 33 \\
\hline $\mathrm{V}-\mathrm{TiO}_{2}$ & 90 & 450 & 1.5 & 1.6 & 66 & $2: 4$ & 2.7 & 20 & 30 & 26 \\
\hline $\mathrm{V}-\mathrm{TiO}_{2}$ & 90 & 450 & 1.5 & 1.6 & 66 & $8: 1$ & 0.7 & 3 & 56 & 54 \\
\hline
\end{tabular}

The proposed reaction mechanisms also indicate that vanadium may change between its oxidation states III, IV, and V. Electron paramagnetic resonance (EPR) is an important analytical method to directly identify different oxidation states in a bulk sample, which might be generated by electronic crossover during the reaction cycle. In particular, it may provide information about the vanadium oxidation states IV and III, both being paramagnetic caused by unpaired d electrons, while vanadi$\mathrm{um}(\mathrm{V})$ is EPR silent. Although vanadium(IV) in its spin doublet state can be detected by standard X-band (9-10 GHz) EPR, vanadium(III) in its spin triplet state probably has a large fine structure (FS) interaction, thus preventing its detection by standard EPR. Other methods for identification of vanadium(III), like X-ray photoelectron spectroscopy (XPS), are mostly applied under ultra-high-vacuum conditions, which may lead to changes in the catalyst structure. The interpretation of such experiments might therefore be erroneous. Because of its characteristic hyperfine structure, the spin doublet state of vanadium(IV) is easily identified at X-band, thus at least providing information about one of the oxidation states. However, an unambiguous interpretation of these spectra is impeded by the fact that several other paramagnetic species, generated during the reaction or even present in the pristine catalyst, lead to crowded spectra which are difficult to unravel because small differences in $g$ values are not sufficient to separate spectra when using the standard external field of $0.3 \mathrm{~T}$.

Application of high-frequency EPR (HF-EPR) in the study is based on a major technological development of EPR in the past decades, not only introducing Fourier-transform (FT) and multipulse EPR as important analytical tools but also expanding its frequency range well above $100 \mathrm{GHz}$ (W-band), which has been commercially available since 1995. At these elevated frequencies the tiny dimensions of microwave resonators restrict sample dimensions to the submillimeter range and, more important, exclude investigation of materials with significant microwave losses. As an alternative, for more general applications a resonator-free set up invoking transmission or reflection like in ordinary optical spectroscopy is the method of choice, in particular if one is not restricted by sample mass. The inherent loss in sensitivity can partially be compensated for using sample dimensions adapted to the cross section of microwave propagation. Successful application of high-frequency EPR for investigation of ferroelectric material was demonstrated recently by our group. ${ }^{16}$ Further examples for use of HF-EPR were compiled in a special issue of Magnetic Resonance in Chemistry. ${ }^{17} \mathrm{We}$ therefore anticipated that this technique could also be applied for the study of catalytically active materials, invoking vanadium as active centers. As shown recently, vanadium(III) can be observed using HF-EPR, leading to a full characterization of its spin Hamiltonian parameters. ${ }^{18}$ We therefore initiated a HFEPR study of various supported vanadium catalysts.

In most previous studies catalysts are characterized before they are used for the respective reaction, ${ }^{7,19,20}$ although it would be much more informative if in situ methods could be invoked. In this study we present results from a pseudo-in-situ approach: The samples were quenched under steady-state reaction conditions by purging the catalyst with nitrogen and cooling it down under inert atmosphere. Subsequently, the catalysts were refilled in glass vials and sealed under high-vacuum conditions, 
preventing any air or moisture contact with the samples. A similar procedure was already described in a study by Chary et al. ${ }^{21}$

\section{Experimental Methods}

2.1. High-Frequency EPR Spectrometer. The highfrequency EPR measurements were performed at the National High Magnetic Field Laboratory (NHMFL), Tallahassee. ${ }^{22}$ The setup used operates in transmission mode and employs oversized cylindrical waveguides. The microwaves were generated by a phase-locked Virginia Diodes source generating a frequency of $13 \pm 1 \mathrm{GHz}$ and producing its harmonics of which the 4th, 8th, 16th, 24th, and 32nd were available. A frequency range of ca. $48-448 \mathrm{GHz}$ can be covered. Microwave power incident on the waveguide was adjusted to approximately $5 \mathrm{~mW}$. Microwave power incident on the sample probably will be on the order of $1 \mathrm{~mW}$. No resonator was used. Microwave detection was performed with a low-noise, fast-response InSb hot-electron bolometer (QMC Ltd.), operated at liquid helium temperature. A field modulation in the range of $10-50 \mathrm{kHz}$ was used to obtain "first-derivative-type" EPR spectra. Microwave frequencies in the range of $200-400 \mathrm{GHz}$ were chosen for our experiments to allow for optimal spectral dispersion and frequency resolution. A superconducting magnet (Oxford Instruments) capable of reaching a field of $14.9 \mathrm{~T}$ was employed, thus covering the important $g=2$ region of spectra, characteristic for doublet radicals.

2.2. Catalyst Preparation. The $\mathrm{Al}_{2} \mathrm{O}_{3}$ - and $\mathrm{TiO}_{2}$-supported catalysts were prepared by saturation wetness impregnation. The chosen support materials were alumina (Alfa Aesar) and titania (Sachtleben Chemie) received as porous pellets. Prior to impregnation, pellets were crushed and sieved to a particle size fraction of $0.1-0.3 \mathrm{~mm}$. A saturated solution of vanadyl acetylacetonate (Sigma-Aldrich, $>97 \%$ ) in toluene was heated under reflux until the boiling point was reached. Subsequently, for each catalyst sample about $2 \mathrm{~g}$ of the support was added to $250 \mathrm{~mL}$ of the mixture and boiled under reflux for about $1 \mathrm{~h}$. The impregnated particles were thoroughly washed with fresh toluene to remove unbound vanadyl species, then dried at 353 $\mathrm{K}$, and finally calcined in air at $773 \mathrm{~K}$ for $3 \mathrm{~h}$. The calcined catalysts were sieved again.

In the case of SBA-15 as a support material a grafting/ionexchange method ${ }^{23}$ was used for this EPR study because samples prepared by this method had been characterized in detail before in our group..$^{9,24}$ The grafting/ion-exchange procedure consists of (1) surface functionalization of SBA-15 using 3-aminopropyltrimethoxysilane (APTMS) and subsequent $\mathrm{HCl}$ treatment leading to formation of the corresponding ammonium salt (functionalized SBA-15), (2) ion exchange of decavanadate, and (3) a final calcination step at $550{ }^{\circ} \mathrm{C}$. Details of the synthesis procedure and its characterization can be found elsewhere..$^{23,25}$ For the catalytic material used here (V-SBA-15) $73 \mathrm{mg}$ of ammonium decavanadate was added to a suspension of $1 \mathrm{~g}$ of functionalized SBA-15 in water.

2.3. Catalytic Measurements. Experiments were carried out at temperatures between 673 and $773 \mathrm{~K}$ using u-shaped fixed bed quartz reactors at atmospheric pressure. For each run, catalyst amounts of 250,150, and $90 \mathrm{mg}$ were used for V-SBA$15, \mathrm{~V}-\mathrm{Al}_{2} \mathrm{O}_{3}$, and $\mathrm{V}-\mathrm{TiO}_{2}$, respectively. Different amounts of catalysts were used in order to achieve comparable degrees of reactant conversion. Using synthetic air as the oxygen source, propane and oxygen were fed in a ratio of $8: 1\left(\mathrm{C}_{3} \mathrm{H}_{8} / \mathrm{O}_{2} / \mathrm{N}_{2}=\right.$ 62.3/7.8/31.2) and 2:4 $\left(\mathrm{C}_{3} \mathrm{H}_{8} / \mathrm{O}_{2} / \mathrm{N}_{2}=9.2 / 18.4 / 73.7\right)$ with a gas hourly space velocity (GHSV) of $5 \times 10^{3}-10^{4} \mathrm{~h}^{-1}$. The experimental setup including product analysis is described in detail elsewhere. ${ }^{26}$

2.4. Parameter Determination. Propane and oxygen conversion $X$ and propene selectivity $S$ as a function of the respective concentration $c_{i}$ were calculated from eqs 1,2 , and 3

$$
\begin{gathered}
X_{\mathrm{C}_{3} \mathrm{H}_{8}}=1-\frac{C_{\mathrm{C}_{3} \mathrm{H}_{8}}}{C_{0, \mathrm{C}_{3} \mathrm{H}_{8}}} \\
X_{\mathrm{O}_{2}}=1 \frac{C_{\mathrm{O}_{2}}}{C_{0, \mathrm{O}_{2}}} \\
S_{\mathrm{C}_{3} \mathrm{H}_{6}}=\frac{C_{\mathrm{C}_{3} \mathrm{H}_{6}}}{C_{0, \mathrm{C}_{3} \mathrm{H}_{6}}-C_{\mathrm{C}_{3} \mathrm{H}_{6}}}
\end{gathered}
$$

\section{Results}

All investigated catalysts have been characterized before. The results were reported in refs 9,11 , and 24 . The catalysts were found to expose highly dispersed vanadium oxide surface species. Both isolated and polymeric vanadium oxide species are present. The $\mathrm{V}-\mathrm{Al}_{2} \mathrm{O}_{3}$ catalyst sample contained negligible amounts of $\mathrm{V}_{2} \mathrm{O}_{5}$ as evaluated on the basis of results from Raman spectroscopy. The loadings, surface areas, and vanadium densities of the samples as well as reaction conditions are summarized in Table 1 . The vanadium oxide loadings (in wt $\%$ $\mathrm{V}_{2} \mathrm{O}_{5}$ ) were in the range of $1.6-4.7 \mathrm{wt} \%$, resulting in surface densities of 0.7 for $\mathrm{V}-\mathrm{SBA}-15$ and about $1.5 \mathrm{~V} \mathrm{~nm}{ }^{-2}$ for $\mathrm{V}-\mathrm{Al}_{2} \mathrm{O}_{3}$ and $\mathrm{V}-\mathrm{TiO}_{2}$. It was shown in previous studies that in the case of $\mathrm{V}-\mathrm{SBA}-15$ no significant effects of vanadium loading on the structure of vanadium sites was observed for low vanadium loadings $\left(<2.3 \mathrm{~V} \mathrm{~nm}^{-2}\right) .^{24}$

In the case of the $\mathrm{V}-\mathrm{SBA}-15$ sample a higher reaction temperature was applied in order to reach comparable degrees of propane conversion at concurrent homogeneity of the catalyst part. The differences in the selectivity-conversion behavior at equal reaction conditions have been discussed in detail before. ${ }^{11}$ The results of this study show that there is a strong influence of the support material on the catalyst activity and product selectivity. The selectivity at a given propane conversion decreases in the order $\mathrm{V}-\mathrm{Al}_{2} \mathrm{O}_{3}>\mathrm{V}-\mathrm{TiO}_{2}>\mathrm{V}-\mathrm{SiO}_{2}$.

In the following, EPR spectra of these catalyst materials before and after reaction will be shown. For all samples studied spectra with a signal-to-noise ratio better than 20 could be obtained with a single sweep of approximately $30 \mathrm{~min}$ duration. Except for field sweeps covering the full range from 0 to $15 \mathrm{~T}$, a linear baseline correction was sufficient, thus allowing reliable numerical integration of the field derivative spectra without truncation of data. All EPR spectra are depicted after integrating the field-modulated data, thus showing the EPR absorption pattern. This is a prerequisite if attempting to compare relative intensities of spectral components of different line width. This was most important for interpretation of the $\mathrm{TiO}_{2}$-based samples because structures differing by more than 2 orders of magnitude difference in line width were present in this sample. As an example, the original field-modulated EPR spectrum of $\mathrm{V}-\mathrm{TiO}_{2}$ with its familiar first-derivative line shape is compared with its single- and double-integrated form in Figure 3. Whereas the original spectrum is completely dominated by the narrow component, the integrated absorption spectrum exhibits an additional broad component, which clearly dominates the EPR spin susceptibility as seen after the second integration.

Figure 4 shows the EPR spectra of bare support materials and catalysts after calcination under ambient conditions. 


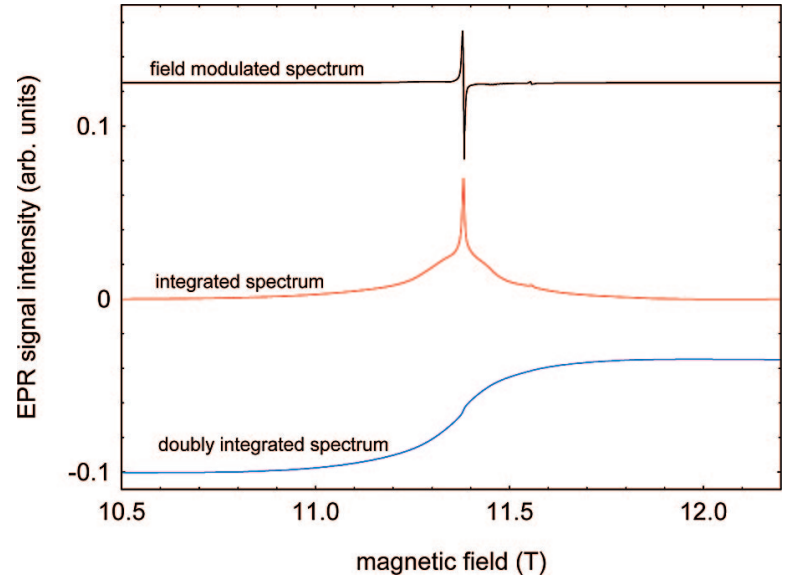

Figure 3. Comparison of the (original) field-modulated $319.2 \mathrm{GHz}$ EPR spectrum of $\mathrm{V}-\mathrm{TiO}_{2}$ with its single and doubly integrated data.

All EPR spectra shown in Figure 4 were measured using approximately the same amount of material. The spectra are depicted after integrating the field-modulated data, thus showing the EPR absorption pattern. As stated above, the number of paramagnetic centers, which is proportional to the EPR susceptibility, can only be obtained by performing a second integration. To facilitate the interpretation, the data are shown by invoking a $g$ value axis instead of the more familiar field dependence. This is equivalent to use of a chemical shift axis in NMR and allows comparing spectra measured with different microwave frequencies. (The direction of the $g$ value axis is inverted to facilitate comparison with spectra presented as conventional field plots).

The resonance field position of all signals was found to be proportional to the microwave frequency used, thus proving assignment to spin doublet radicals $\left(S_{\text {eff }}=1 / 2\right)$. All spectra of the bare support materials show signals in the region of $g=2$, close to the free-electron value. The intensities of these signals at $g=2$ are increasing in the order SBA-15< $\mathrm{Al}_{2} \mathrm{O}_{3} \approx \mathrm{TiO}_{2}$. Whereas only a single component can be identified for $\mathrm{A}$ and $\mathrm{B}$ in the $g=2$ region, at least two components also centered at $g=2$ but differing in $g$ spread can be identified for $\mathrm{TiO}_{2}$. A small peak can be seen at $g=1.982$ for $\mathrm{Al}_{2} \mathrm{O}_{3}$ and $\mathrm{TiO}_{2}$. The bare SBA-15 support material is nearly free of paramagnetic centers (A). The spectral change after coating allows an unambiguous assignment of the signal observed for V-SBA15 as related to vanadium(IV) in its spin doublet state. The powder pattern can be fitted with a $g$ matrix of nearly axial symmetry with $g_{1}=1.98077(2), g_{2}=1.97380(2)$, and $g_{3}=$ $1.93300(2)$ in combination with a vanadium hyperfine tensor with elements 104(2), 64(5), and 550(10) MHz. In particular, the large hyperfine interaction in the "parallel" orientation with $I=7 / 2$ spin is characteristic for vanadium(IV). ${ }^{27}$ In the case of $\mathrm{V}-\mathrm{Al}_{2} \mathrm{O}_{3}$, the vanadium(IV) signal can also be identified on top of a broad background signal, which is present already in the uncoated alumina support material. It is noteworthy that a signal which could be attributed to vanadium(IV) with its characteristic spin parameters as listed above is completely absent in the case of $\mathrm{V}-\mathrm{TiO}_{2}$. The narrow spike at approximately $g=1.97$ cannot be assigned yet.

Figure 5 illustrates spectra of sealed samples of V-SBA-15, $\mathrm{V}-\mathrm{Al}_{2} \mathrm{O}_{3}$, and $\mathrm{V}-\mathrm{TiO}_{2}$ after being used as catalysts using propane and oxygen in a ratio of $8: 1$. The emerging spectra clearly depend on the support material. Signal intensities cannot be compared considering that the V-SBA-15 signal was
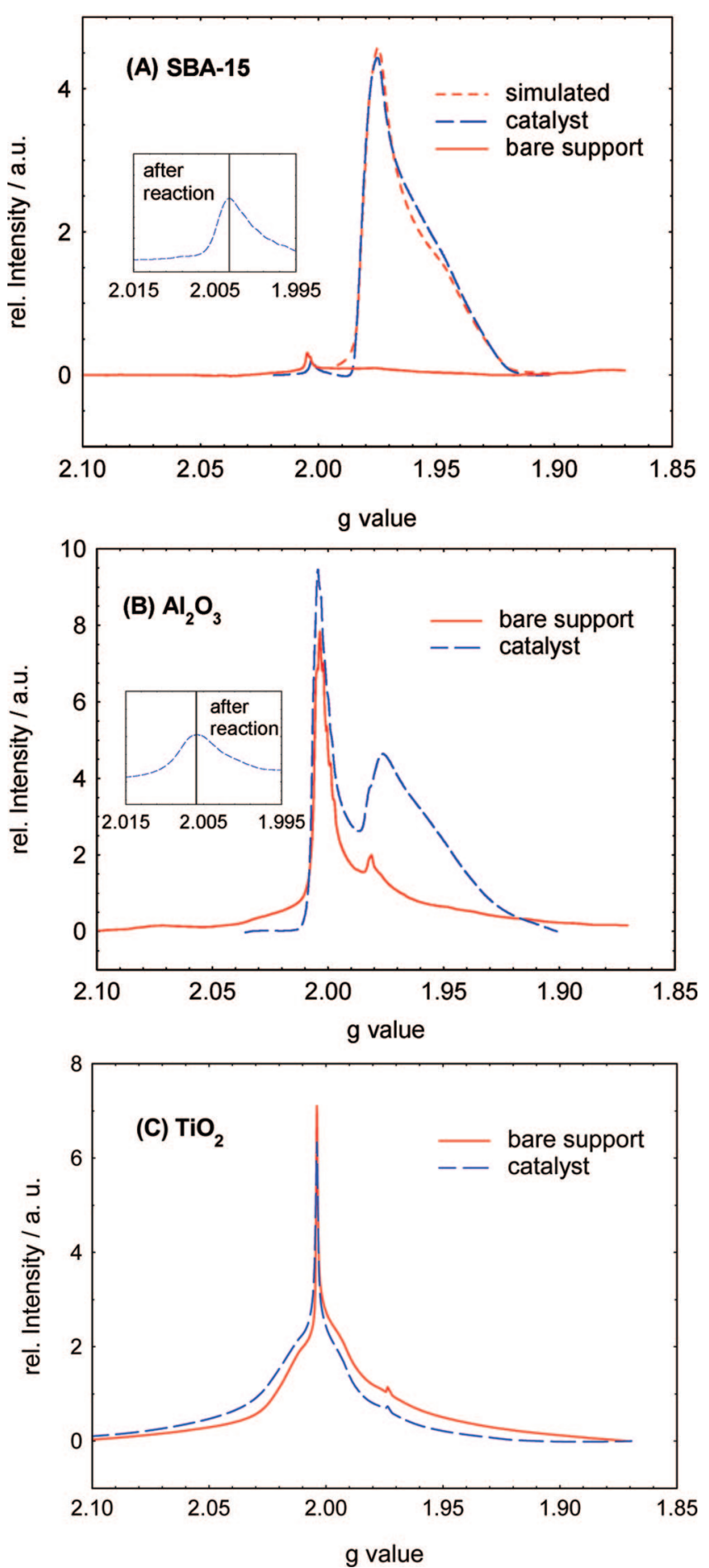

Figure 4. HF-EPR spectra $(319.2 \mathrm{GHz}, 20 \mathrm{~K})$ of the bare support material and respective catalyst (A) SBA-15/V-SBA-15, (B) $\mathrm{Al}_{2} \mathrm{O}_{3} /$ $\mathrm{V}-\mathrm{Al}_{2} \mathrm{O}_{3}$, (C) $\mathrm{TiO}_{2} / \mathrm{V}-\mathrm{TiO}_{2}$ "as is" after calcination. Experimental conditions used for all spectra were kept equal, thus allowing for comparison of the relative intensities of $\mathrm{A}-\mathrm{C}$. The simulation of the vanadium(IV) spectrum shown in A was performed using $g$ matrix and vanadium hyperfine tensor values given in the text. Inserts with increased $g$ resolution indicate the position of signals close to $g=2$. Weak signals originating from manganese(II) impurities are also visible.

multiplied by a factor of 10 in the figure. For $\mathrm{V}-\mathrm{TiO}_{2}$ a new signal emerged at $g=1.935$, which is assigned to titanium(III) (see discussion below).

In Figure 6A, 6B, and 6C the influence of different reaction conditions is shown separately for all three support materials. 


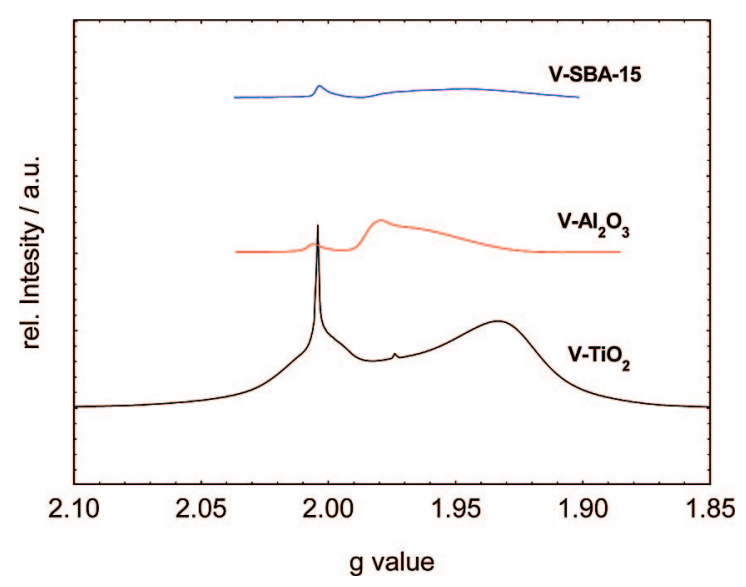

Figure 5. EPR spectra $(319.2 \mathrm{GHz}, 20 \mathrm{~K})$ of sealed catalyst samples in their pseudo-in-situ condition, after being exposed to propane and oxygen in a ratio of (A) 8:1 and (B) 2:4 under reaction conditions.

In the case of $\mathrm{V}-\mathrm{SBA}-15$ (Figure 6A) it can be seen that the signal intensity of the signal at $g=2.0039$ increases by an order of magnitude. The signal intensities can be directly compared because the differences in sample mass have been used to scale the EPR data. Since these signals are observed only after exposing to propane and considering the very small $g$ shift from the free electron value, we assign this signal to carbon-centered radicals on the surface of V-SBA-15. Such radicals are a consequence of cracking reactions occurring at elevated temperatures. The powder pattern of vanadium(IV) is slightly changing, and the apparent peak maximum is decreasing from $g=1.976$ for the fresh sample to $g=1.96$ and 1.955 for the 2:4 and 8:1 samples, respectively.

For $\mathrm{V}-\mathrm{Al}_{2} \mathrm{O}_{3}$ (Figure 6B) the intensity of the signal at $g=$ 2.0062(3) is nearly constant for fresh and pseudo-in-situ samples. The signal apparently originates from paramagnetic centers of the bulk material, which are not influenced by reactions on the surface. This indicates that they are not mobile under typical high-temperature reaction conditions. The significant upshift of $g$ from the free-electron value suggests that these signals originate from oxygen vacancies. After reaction, a shoulder develops at a slightly smaller $g$ value. Spectral resolution, however, is not sufficient to confirm the anticipated presence of additional carbon-centered radicals. Signal intensities attributed to vanadium(IV) increase under oxygen-deficient reaction conditions. Compared to the well-defined pattern observed for the unreacted V-SBA-15 catalyst, it is obvious that a distribution of different vanadium(IV) sites is present, their relative intensity being influenced by the reaction.

In the case of $\mathrm{V}-\mathrm{TiO}_{2}$ (Figure $6 \mathrm{C}$ ), the striking change in the spectrum caused by exposing the material to reaction conditions is the appearance of a strong signal with a new peak at $g=1.93$. Considering the different $g$ value and lack of $g$ anisotropy characteristic for the vanadium(IV) species, we assign this signal to titanium(III) instead (vide infra). The intensity of the spectral features close to $g=2$ is also increasing, which may also be a consequence of formation of carbon-centered radicals. A detailed discussion of the signal consisting of at least two components is given below.

A direct test for the anticipated possibility to detect weak triplet state signals with the experimental setup used is given in Figure 7. This graph shows the simulated and experimentally detected signals of triplet state molecular oxygen, adsorbed on the surface of the open, unreacted sample. The weak signals seen only for the "open" sample can unambiguously be assigned
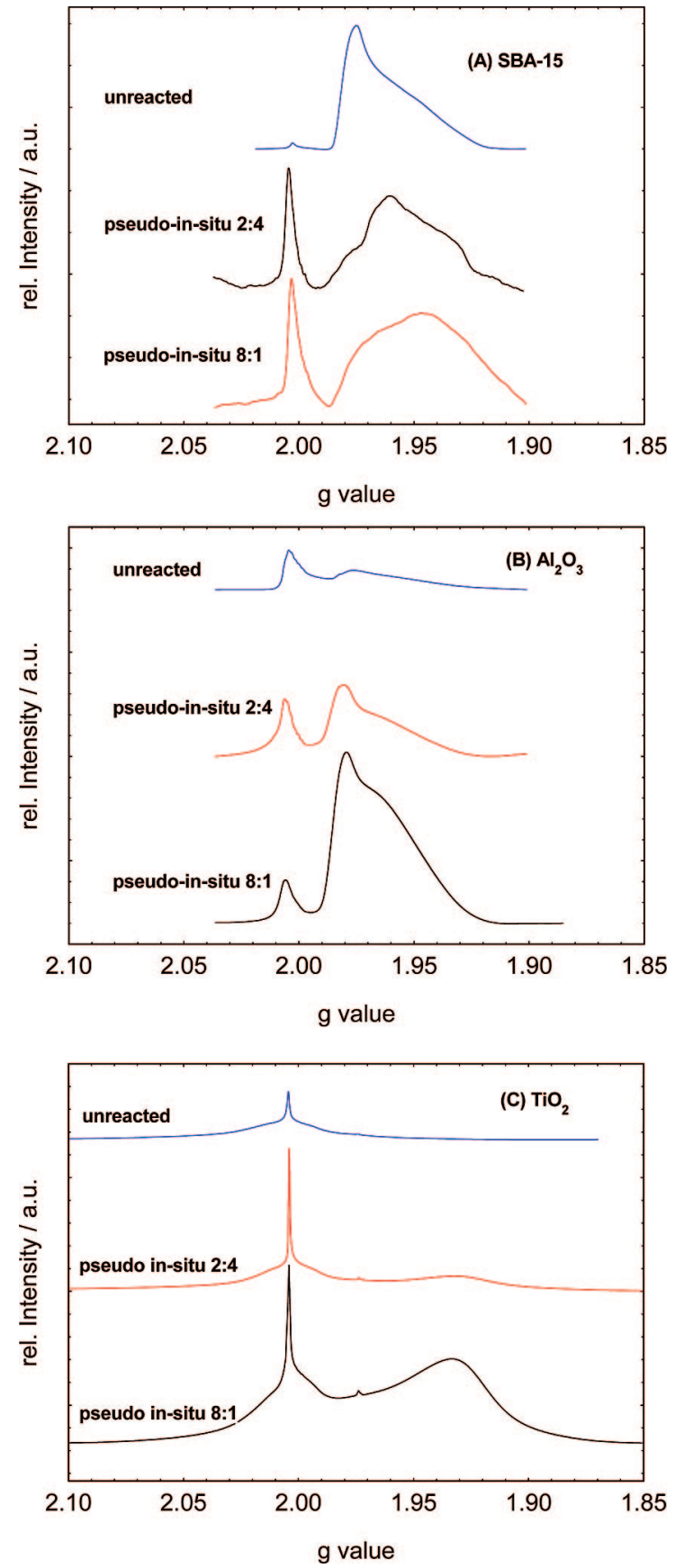

Figure 6. EPR spectra $(319.2 \mathrm{GHz}, 20 \mathrm{~K})$ of unreacted and reacted catalysts, after being exposed to propane and oxygen in a ratio of $8: 1$ and 2:4: (A) V-SBA-15, (B) $\mathrm{V}-\mathrm{Al}_{2} \mathrm{O}_{3},(\mathrm{C}) \mathrm{V}-\mathrm{TiO}_{2}$.

to molecular oxygen. For spectral simulation a ZFS constant of $106.4 \mathrm{GHz}$ was used.

A search for signals assigned to vanadium(III) was unsuccessful for all samples studied. In contrast to EPR spectra of doublet state paramagnetic centers, for which the dominant term in the spin Hamiltonian is given by the Zeeman interaction, the leading term in the spin Hamiltonian of triplet state compounds can be the fine structure interaction, usually termed "zero-field splitting" interaction. ${ }^{28}$ Whereas for powder samples the spectral width in the case of $S=1 / 2$ centers is determined by the $g$ matrix anisotropy, the spread of absorption signals of $S=1$ spectra might be dominated by ZFS and broadened beyond detection limits. In contrast, the position of formally "forbidden" $\Delta m_{\mathrm{S}}= \pm 2$ transitions are invariant (in first order) with respect to orientation, and therefore, these lines are most prominent in 


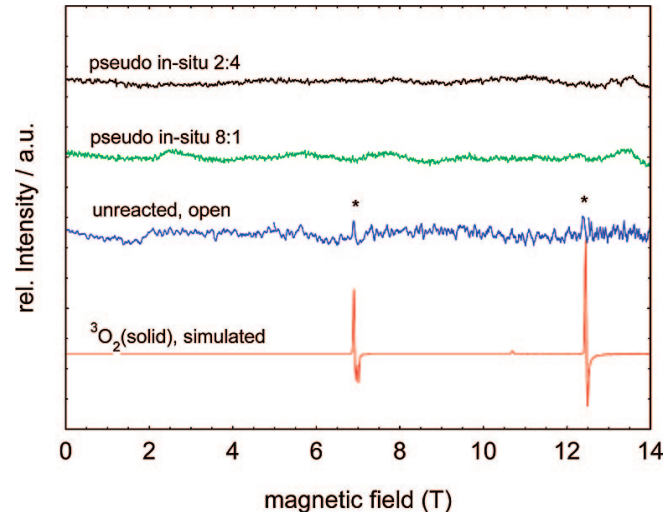

Figure 7. Full-range experimental EPR spectra $(319.4 \mathrm{GHz}, T=20$ $\mathrm{K}$, respective signals marked with an asterisk) of sealed and open catalyst samples (alumina support) in comparison with a simulated ${ }^{3} \mathrm{O}_{2}$ spectrum.

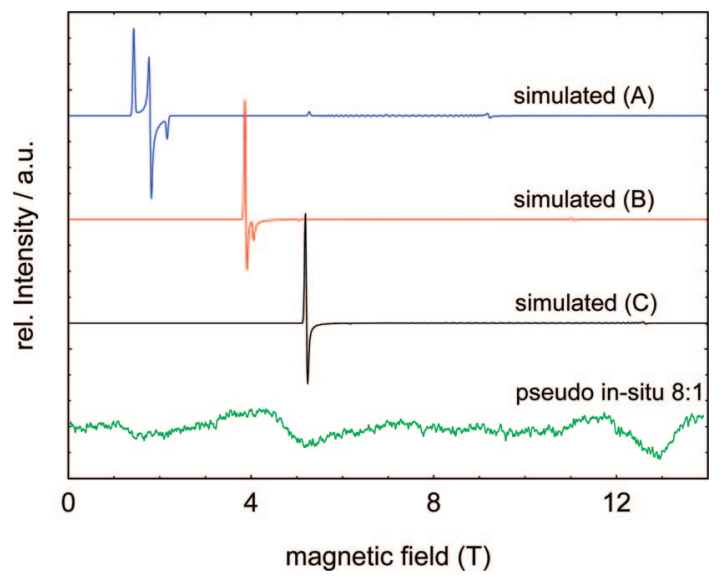

Figure 8. Experimental and simulated EPR powder spectra of a fictitious vanadium(III) center with (A) $D=336 \mathrm{GHz}, E=57.4 \mathrm{GHz}$, (B) $D=280, E=57.4 \mathrm{GHz}$, and (C) $D=224, E=57.4 \mathrm{GHz}$. The microwave frequency used in the experiment and for simulation was $406.4 \mathrm{GHz}$.

powder samples. Figure 8 shows the simulated triplet state powder spectra based on typical values for the ZFS splitting of vanadium(III) centers with low site symmetry. ${ }^{18}$ Whereas for ZFS of "medium" size the signals are observed close to $g=4$ ("half-field condition", here $B=7.1 \mathrm{~T}$ ), increasing ZFS values leads to a down shift of the signals until they are no longer observable. The lack of observation of these lines for the catalyst samples using a microwave frequency of up to $406 \mathrm{GHz}$ puts a lower limit of $340 \mathrm{GHz}$ for the ZFS constant of potentially present vanadium(III) centers.

\section{Discussion}

The focus of this study was the impact of the support material on formation and modification of paramagnetic centers during catalytic reaction. Because of the enhanced resolution of HFEPR signals on a $g$-value scale, spectral components around $g$ $=2$ and those in the range below 1.98 can be separated. In this low- $g$ value range, signals from vanadium(IV) and titanium(III) are expected. In the region of smaller $g$ values, signals which can be attributed to vanadium(IV) are observed only when using SBA-15 and $\mathrm{Al}_{2} \mathrm{O}_{3}$ as support. This assignment is based on the characteristic powder pattern most clearly seen for the V-SBA15 material. The spectrum of the unreacted sample can be fitted with a $g$ matrix of near axial symmetry and a large, unresolved vanadium hyperfine interaction. Fit parameters $(g=1.98077$,
1.97380, and 1.93300 and $A=104,64$, and $550 \mathrm{MHz}$ ) are both typical for vanadium(IV). In the case of V-SBA-15, the apparent peak maxima of vanadium(IV), corresponding to the perpendicular orientation, are decreasing from $g=1.976$ for the fresh sample to $g=1.96$ and 1.955 for the reacted sample. This can be attributed to the fact that the well-defined local site symmetry of vanadium(IV) on the surface of the fresh catalyst is modified by generation of additional paramagnetic centers and probably also by deposition of diamagnetic reaction products. The characteristic EPR signature of vanadium(IV) is preserved however. In the case of the alumina-supported catalyst we also observe an increase in signal intensity of the characteristic vanadium(IV) pattern under oxygen-lean conditions. Because of the underlying broad background signal already present in the pure support material and in the fresh, unreacted $\mathrm{V}-\mathrm{Al}_{2} \mathrm{O}_{3}$ sample, a spectral fit of vanadium(IV) was not attempted. However, comparison with the V-SBA-15 spectra indicates that a distribution of sites with different local symmetries is generated.

A striking difference in EPR pattern is observed for the $\mathrm{TiO}_{2}$ support material before as well as after reaction. A strong unstructured signal with a maximum at $g=1.93$ is created by the catalytic reaction, being completely absent for the other support materials. Previous EPR studies on $\mathrm{TiO}_{2}$ in its anatase and rutile phases have revealed unique local sites for lattice-trapped electrons in the bulk material, resulting in well-defined values for the $g$ matrix of such titanium(III) centers $\left(g^{\text {(anatase) }}\right.$ perpendicular $=1.976, g^{\text {(anatase) }}$ parallel $\left.=1.990 ; g^{\text {(rutile) }}{ }_{\text {perpendicular }}=1.976, g^{\text {(rutile) }}{ }_{\text {parallel }}=1.941^{29}\right)$. For surface-trapped electrons, however, a broad signal with a peak at $g=1.93$ was observed instead. ${ }^{30}$ A similar pattern was also observed for surface titanium(III) cations in a tetragonally distorted environment in different matrices such as oxide of fluoride glasses. ${ }^{31}$ The observed $g$ value of 1.933 in our sample would be consistent with the assumption that electrons trapped at titanium(IV) sites on the surface during the catalytic reaction give rise to the EPR signal, which is specific for the $\mathrm{TiO}_{2}$-supported catalyst. The wide distribution of $g$ values leading to the broad signal is indicative of a large variation of local symmetries. This assignment is further supported by our recent observation that the peak maximum of this characteristic signal is shifted from $g=1.933$ to 1.965 if methanol instead of propane is used as a substrate. This shift is explained by considering the partial substitution of oxygen anions by hydroxyl groups. ${ }^{32}$ The remaining spectral properties centered at $g=2$ were not affected, even when changing the substrate.

Doublet radicals with a $g$ value close to the free-electron value are either already present for the pure support material or generated during the reaction. The nature of the signals seen for the SBA-15 and $\mathrm{Al}_{2} \mathrm{O}_{3}$ support close to $g=2$ is yet unknown. Using HF EPR with its inherent increase in $g$ resolution it was possible to identify unambiguously a difference in $g$ values for these radicals on the two support materials. Whereas the small positive $g$ shift of +0.0016 with respect to the free electron value of 2.002319 seen for V-SBA-15 after reaction is indicative for carbon-centered radicals, the larger $g$ shift of +0.0039 found for the alumina-supported catalyst even before catalytic reaction indicates partial spin localization at oxygen, also referred to as "oxoradical", with its increased spin-orbit coupling in the $\mathrm{Al}_{2} \mathrm{O}_{3}$ lattice.

The pure $\mathrm{TiO}_{2}$ support material as well as the catalyst before and after reaction exhibit a narrow line superimposed on a broad signal close to $g=2$ with line width parameters completely 
different from the pattern observed for SBA-15 and alumina. It should be noted that the total spin susceptibility, i.e., the number of paramagnetic centers derived from the broader component, is larger by a factor 10 than that from the narrow line (see Figure $3)$. The spectral pattern is nearly invariant, but the signal intensity is significantly larger after the samples were exposed to reaction conditions. These $g=2$ centers are tentatively ascribed to oxygen vacancies in the bulk support material and paramagnetic centers on the surface. This assignment is supported by a recent EPR study of titania anatase nanoparticles, giving evidence for trapped electrons on oxygen vacancies. ${ }^{33}$ The $g$ value reported for these F centers $(g=2.0034)$ are close to the $g$ value of the narrow component $(g=2.0043(4))$. The dominant broader component exhibits an asymmetric powder line shape, which is more pronounced when taking spectra with the highest available microwave frequency $406.4 \mathrm{GHz}$. Although not fully resolved, the spectrum can be described with an anisotropic $g$ matrix $\left(g_{1}=2.027(25), g_{2}=2.011(15), g_{3}=\right.$ 1.992(6)). This set is similar to the values reported for $\mathrm{O}_{2}{ }^{-}$ absorbed on the surface of anatase particles. ${ }^{34}$ Thus, it seems possible that electrons can be trapped at titanium(IV) as well as on surface-bound molecular oxygen on the titania surface, significantly influencing the catalytic redox cycle and/or leading to additional unselective reaction pathways. It can be excluded that the broad component results from $\mathrm{O}_{2}{ }^{(-)}$radicals directly coordinated to vanadium because the signal is already present before coating the support material. The additional presence of bulk oxygen vacancies which probably are mobile at elevated temperatures might also influence the catalytic behavior, although their relative abundance is small.

A recent study by Ganduglia-Pirovano et al. ${ }^{35}$ identified oxygen vacancies as being possibly involved in catalytic processes during oxidative dehydrogenation reactions. In addition, a recent study of Goodrow et al. ${ }^{36}$ investigated the influence of oxygen vacancies on the methanol oxidation on titaniasupported catalysts by means of DFT calculations. They came to the conclusion that oxygen vacancies are part of the active center and serve to reduce the activation energy of the ratedetermining step for methanol oxidation. Both studies indicate that formation of oxygen vacancies could have a major influence on the catalytic performance of oxidative dehydrogenation reactions. An interesting aspect is that except for SBA-15, oxygen vacancies already exist in the bare support materials chosen for this study. Sekiya et al. ${ }^{37}$ studied the behavior of oxygen vacancies in $\mathrm{TiO}_{2}$ single crystals (anatase) and found that they can be even stable under oxidizing conditions at elevated temperatures. This would explain the abundance of oxygen vacancies in the pseudo-in-situ samples, which were exposed to high oxygen partial pressures.

The absence of persistent vanadium(III) signals in all samples does not exclude such sites being involved in ODP. As extremely short-lived catalytic species they might generally evade detection by "postreaction" EPR as long as oxygen is present in the reaction mixture. This seems generally to be the case because even under oxygen lean conditions more than $2 \%$ of oxygen is left in the reaction mixture. Evidence for vanadium(III) surface species has been presented in previous XPS studies on the basis of a V $2 \mathrm{p}_{3 / 2}$ band around $515.9 \mathrm{eV} .{ }^{24,38}$ However, recent time-dependent XPS experiments, which eliminate the influence of vacuum/X-ray radiation by extrapolating the temporal changes to $t=0$, indicate the absence (contribution $\sim 1 \%$ ) of vanadium(III) in the case of dehydrated (hydrated) silica-supported vanadia samples. ${ }^{24}$

\section{Conclusions}

Application of HF-EPR for investigation of catalysts was found to be very promising because of the significant improvement of spectral resolution. At microwave frequencies of 300 $\mathrm{GHz}$ and above with the corresponding magnetic fields in the range of $10-15 \mathrm{~T}$ it is possible to separate spectral components originating from various transition-metal ions from carboncentered radicals or oxygen vacancies. High sensitivity for detection of paramagnetic centers is provided at these high frequencies because nearly complete spin polarization is obtained if spectra are taken below $20 \mathrm{~K}$. This allows performing experiments without the use of a resonance cavity. EPR in transmission mode imposes much less restrictions on sample dimensions and dielectric properties and can thus be invoked to study catalysts because in general the amount of available samples is not restricted. It was possible to investigate probes extracted from the reactor under "inert" conditions, sealed in quartz sample tubes of $6 \mathrm{~mm}$ o.d. and $50 \mathrm{~mm}$ length. Comparison of pure support materials with unreacted catalysts and catalysts exposed to reaction conditions helped to identify paramagnetic centers which might be related to catalytic active sites in ODP.

In this study silica-, alumina-, and titania-supported vanadium oxide catalysts were investigated. Before being used as catalysts, one well-defined vanadium(IV) surface site was found in the case of the SBA-15 support and a variety of different vanadium(IV) sites in the case of the alumina-supported material. No vanadium(IV) centers are detected in the case of the titaniasupported catalyst. For this material strong signals originating from $\mathrm{F}$ centers, indicating the presence of oxygen vacancies, and, more abundant, from surface-trapped $\mathrm{O}_{2}{ }^{-}$radicals are also observed. After being exposed to reaction conditions, additional signals are detected due to the presence of carbon-centered radicals for SBA-15 and partial electron localization on the alumina lattice (oxoradicals) in the case of alumina-supported catalysts. In contrast, a signal attributed to surface-trapped electrons forming titanium(III) centers is generated by the catalytic reaction of $\mathrm{TiO}_{2}$-supported samples. The number of $\mathrm{F}$ centers and trapped $\mathrm{O}_{2}{ }^{-}$radicals increased during reaction of the titania-supported catalyst. We therefore conclude that the reaction scheme depicted in Figure 2 has to be appended by including the electronic properties of the support materials. The suggestion of Chen et al. ${ }^{14}$ relating catalytic activity to the extent of electron delocalization on a given catalyst could be extended by predictions about the different mechanisms of such delocalization on the respective support material as evident from the creation of persistent radicals. Still one has to keep in mind that all catalysts were investigated under post reaction conditions and relevant intermediate states might have evaded detection.

No indication of persistent vanadium(III) could be found in any sample, although they could be present as short-lived species during the catalytic reaction. However, a reaction mechanism of ODP without participation of vanadium(III) is generally possible for supported isolated and associated vanadium oxide species as shown in Figure 2 and predicted to be energetically preferred by DFT calculations.

From our study there is evidence that titanium as a support material for vanadium oxide is involved in the key steps of ODP by trapping electrons either on titanium(IV) surface states or on surface-trapped molecular oxygen. In this context it will be of interest if similar changes in the EPR spectra are observed when exposing the bare $\mathrm{TiO}_{2}$ support material to reaction conditions. The results indicate that alumina participates in mechanisms of electron delocalization during catalytic turnovers 
through partial localization of electrons on oxo radicals. No participation of the support material on the catalytic reaction was found for the SBA-15-supported catalysts. In addition, different structural properties of the active site have to be considered. This is suggested by Raman spectra of these catalysts presented in a previous publication. ${ }^{11}$ In order to derive a correlation between persistent reduced states and catalytic performance a quantitative study invoking comparable reaction conditions will be performed in the future.

Acknowledgment. This work was supported by the German Research Foundation (Deutsche Forschungsgemeinschaft, DFG) through the corporate research center "Structure, dynamics and reactivity of transition metal oxide aggregates" (Sonderforschungsbereich 546, http://www.chemie.hu-berlin.de/sfb546). The authors thank Robert Schlögl for discussions and Rita Herbert for preparation of some of the catalytic materials. C.H. thanks the Deutsche Forschungsgemeinschaft (DFG) for providing an Emmy Noether fellowship. K.P.D. is grateful for support from the NHFML during his sabbatical at Tallahassee. A.D. is grateful for helpful discussions with Prof. A. T. Bell (University of California at Berkeley).

\section{References and Notes} 467.

(1) Chaar, M. A.; Patel, D.; Kung, H. H. J. Catal. 1988, 109, 463-

(2) Sam, D. S. H.; Soenen, V.; Volta, J. C. J. Catal. 1990, 123, 417435.

(3) Capannelli, G.; Bottino, A.; Romano, D.; Monticelli, O.; Servida, A.; Cavani, F.; Bartolini, A.; Rossini, S. Nat. Gas Conv. V 1998, 119, 423428.

(4) Gilardoni, F.; Bell, A. T.; Chakraborty, A.; Boulet, P. J. Phys. Chem. $B$ 2000, 104, 12250-12255. 131.

(5) Cavani, F.; Ballarini, N.; Cericola, A. Catal. Today 2007, 127, 113-

(6) Rozanska, X.; Fortrie, R.; Sauer, J. J. Phys. Chem. C 2007, 111, 6041-6050.

(7) Khodakov, A.; Olthof, B.; Bell, A. T.; Iglesia, E. J. Catal. 1999, $181,205-216$.

(8) Weckhuysen, B. M.; Keller, D. E. Catal. Today 2003, 78, 25-46. 1777.

(9) Venkov, T. V.; Hess, C.; Jentoft, F. C. Langmuir 2007, 23, $1768-$

(10) Chen, K. D.; Khodakov, A.; Yang, J.; Bell, A. T.; Iglesia, E. J. Catal. 1999, 186, 325-333.

(11) Dinse, A.; Frank, B.; Hess, C.; Habel, D.; Schomäcker, R. J. Mol. Catal. A 2008, 289, 28-37.

(12) Chen, K. D.; Bell, A. T.; Iglesia, E. J. Phys. Chem. B 2000, 104, 1292-1299.
(13) Chen, K. D.; Iglesia, E.; Bell, A. T. J. Phys. Chem. B 2001, 105, 646-653.

(14) Chen, K. D.; Bell, A. T.; Iglesia, E. J. Catal. 2002, 209, 35-42.

(15) Argyle, M. D.; Chen, K. D.; Bell, A. T.; Iglesia, E. J. Catal. 2002, 208, 139-149.

(16) Mestric, H.; Eichel, R. A.; Kloss, T.; Dinse, K. P.; Laubach, S.; Laubach, S.; Schmidt, P. C.; Schönau, K. A.; Knapp, M.; Ehrenberg, H. Phys. Rev. B 2005, 71, 134109-134119.

(17) Lubitz, W.; Möbius, K.; Dinse, K. P. Magn. Reson. Chem. 2005, $43, \mathrm{~S} 2-\mathrm{S} 3$.

(18) Krzystek, J.; Fiedler, A. T.; Sokol, J. J.; Ozarowski, A.; Zvyagin, S. A.; Brunold, T. C.; Long, J. R.; Brunel, L. C.; Telser, J. Inorg. Chem. 2004, 43, 5645-5658.

(19) Kondratenko, E. V.; Cherian, M.; Baerns, M.; Su, D. S.; Schlögl, R.; Wang, X.; Wachs, I. E. J. Catal. 2005, 234, 131-142.

(20) Blasco, T.; Galli, A.; Nieto, J. M. L.; Trifiro, F. J. Catal. 1997, 169, 203-211.

(21) Chary, K. V. R.; Reddy, B. M.; Nag, N. K.; Subrahmanyam, V. S.; Sunandana, C. S. J. Phys. Chem. 1984, 88, 2622-2624.

(22) Hassan, A. K.; Pardi, L. A.; Krzystek, J.; Sienkiewicz, A.; Goy, P.; Rohrer, M.; Brunel, L. C. J. Magn. Reson. 2000, 142, 300-312.

(23) Hess, C.; Wild, U.; Schlögl, R. Microporous Mesoporous Mater. 2006, 95, 339-349.

(24) Hess, C.; Tzolova-Muller, G.; Herbert, R. J. Phys. Chem. C 2007, $111,9471-9479$.

(25) Hess, C.; Hoefelmeyer, J. D.; Tilley, T. D. J. Phys. Chem. B 2004, 108, 9703-9709.

(26) Frank, B.; Dinse, A.; Ovsitser, O.; Kondratenko, E. V.; Schomäcker, R. Appl. Catal., A 2007, 323, 66-76.

(27) Abragam, A.; Bleaney, B. EPR of Transition Ions; Oxford University Press: New York, 1970.

(28) Carrington, A.; McLachlan, A. D. Introduction to Magnetic Resonance with Applications to Chemistry and Chemical Physics; Wiley: New York, 1999.

(29) Hurum, D. C.; Agrios, A. G.; Gray, K. A.; Rajh, T.; Thurnauer, M. C. J. Phys. Chem. B 2003, 107, 4545-4549.

(30) Hurum, D. C.; Gray, K. A.; Rajh, T.; Thurnauer, M. C. J. Phys. Chem. B 2005, 109, 977-980.

(31) Aboukais, A.; Bogomolova, L. D.; Deshkovskaya, A. A.; Jachkin, V. A.; Krasil'nikova, N. A.; Prushinsky, S. A.; Trul, O. A.; Stefanovsky, S. V.; Zhilinskaya, E. A. Opt. Mater. 2002, 19, 295-306.

(32) Coronado, J. M.; Maira, A. J.; Conesa, J. C.; Yeung, K. L.; Augugliaro, V.; Soria, J. Langmuir 2001, 17, 5368-5374.

(33) Sun, Y.; Egawa, T.; Zhang, L. Y.; Yao, X. J. Mater. Sci. Lett. 2003, 22, 799-802.

(34) Yamashita, H.; Harada, M.; Misaka, J.; Takeuchi, M.; Ikeue, K.; Anpo, M. J. Photochem. Photobiol. A 2002, 148, 257-261.

(35) Ganduglia-Pirovano, M. V.; Hofmann, A.; Sauer, J. Surf. Sci. Rep. 2007, 62, 219-270.

(36) Goodrow, A.; Bell, A. T. J. Phys. Chem. C 2008, 112, 1320413214.

(37) Sekiya, T.; Yagisawa, T.; Kamiya, N.; Das Mulmi, D.; Kurita, S.; Murakami, Y.; Kodaira, T. J. Phys. Soc. Jpn. 2004, 73, 703-710.

(38) Eberhardt, M. A.; Proctor, A.; Houalla, M.; Hercules, D. M. J. Catal. 1996, 160, 27-34.

JP807159F 